\title{
Pathogenesis of kidney disease in systemic lupus erythematosus
}

\author{
Harini Bagavant ${ }^{\mathrm{a}}$ and Shu Man Fu ${ }^{a, b}$ \\ a Division of Nephrology, Center for Immunity, Inflammation and Regenerative Medicine, University \\ of Virginia, School of Medicine, Charlottesville, Virginia, USA \\ b Microbiology, Department of Medicine, University of Virginia, School of Medicine, Charlottesville, \\ Virginia, USA
}

\begin{abstract}
Purpose of review-A combination of systemic autoimmunity and tissue response to immune injury underlie renal involvement in lupus erythematosus. In this review, we discuss recent literature investigating pathogenetic mechanisms of lupus glomerulonephritis.

Recent findings-In lupus glomerulonephritis, glomerular immune complexes were believed to be the primary mediators of renal disease. Recent studies make it apparent that autoantibodies of multiple specificities participate in the formation of immune complexes, deposited in the kidneys. Renal infiltration by T cells, macrophages, and dendritic cells have a dominant role in the progression of lupus glomerulonephritis leading to renal failure. Activation of Toll-like receptors modulates autoantibody production and systemic interferon responses. However, glomerular cell responses to immune injury influence disease outcome. In addition, new insights on the genetics of susceptibility to end-organ damage in lupus glomerulonephritis have been discovered. Differential glomerular responses reflected in gene expression profiles during disease progression provide potential markers for diagnosis of lupus glomerulonephritis progression and flares. In addition, studies of end-organ responses provide new targets for therapeutic interventions.
\end{abstract}

Summary-Lupus glomerulonephritis is a prototype of immune complex disease mediated by autoantibodies of multiple specificities, one of which is anti-DNA. Murine models of spontaneous systemic lupus erythematosus have been critical for understanding the underlying disease. Recent studies demonstrate that in addition to systemic autoimmunity, end-organ responses, and end-organ resistance to damage are also critical in determining disease outcome. This understanding should influence design of novel therapeutic approaches in systemic lupus erythematosus.

\section{Keywords}

autoantibodies; end-organ resistance to damage; glomerular gene expression; lupus nephritis; mouse models; SLE

\section{Introduction}

Systemic lupus erythematosus (SLE) is an autoimmune disorder affecting multiple organ systems including kidney, skin, lung, heart, the hematopoietic system and brain [1].

Glomerulonephritis leading to severe persistent proteinuria, chronic renal failure and end-stage renal disease remains one of the most severe complications of SLE and is associated with significant morbidity and mortality. Despite intensive investigation, the mechanisms underlying renal injury in lupus glomerulonephritis are not completely understood. In this

Correspondence to Dr Shu Man Fu, Department of Medicine, University of Virginia, School of Medicine, Box 800133, Charlottesville, VA 22908, USA, Tel: +1 434924 9627; fax: +1 434924 9578; sf2e@ virginia.edu. 
review, we will discuss recent literature on autoimmune responses and renal factors that influence lupus glomerulonephritis. This article reviews literature on murine models of spontaneous lupus glomerulonephritis including the MRL/lpr, NZB/W F1, NZM2328, NZM2410 and BXSB-Yaa. Certain relevant clinical studies from lupus patients are also discussed.

\section{Pathogenic autoantibodies in lupus glomerulonephritis}

Detection of antinuclear antibodies in serum is one of the most frequent laboratory tests for diagnosis of SLE. Anti-dsDNA antibodies are considered a hallmark of SLE and anti-Sm antibodies have a significant association with lupus nephritis [2,3]. However, not all antidsDNA antibodies can deposit in the kidney, nor has it been possible to replicate the disease of lupus nephritis with passive transfer of anti-DNA antibodies [4]. In mice, ability of antiDNA antibodies to induce glomerulonephritis and transient proteinuria has been attributed to their cross-reactivity to glomerular antigens such as alpha actinin and laminin $[5,6]$. Deposition of positively charged nucleosomes on glomerular basement membrane (GBM) as targets for autoantibodies has been previously described [7]. In a recent series of articles, Rekvig and colleagues $[8,9]$ demonstrate that electron dense deposits are seen in proteinuric, lupussusceptible NZB/W F1 mice. These deposits are oligo-nucleosomes, recognized by antidsDNA reactive monoclonal antibodies, and caused by ineffective fragmentation and clearance of apoptotic material in the renal glomerulus. In addition, the authors provide evidence that the antibodies eluted from the kidneys of nephritic mice have a higher affinity for DNA compared with serum anti-DNA antibodies [10]. These antibodies react specifically with the electron dense deposits and not with surrounding tissues containing alpha actinin or laminin $[11,12]$. It was concluded that the accumulation of chromatin fragments on the glomerular basement membrane and high-affinity antibodies to nucleosomal DNA, not cross-reactivity to glomerular proteins determines pathogenic capacity of anti-DNA antibodies. One caveat in these studies is that the glomerular electron dense deposits were not detectable before 26 weeks of age at which time the mice had severe proteinuria indicative of well established lupus glomerulonephritis. In addition, the monospecificity of the anti-DNA antibodies requires further documentation. Thus, the specificities of autoantibodies in immune complex deposits initiating the disease remain to be determined. Moreover, these results are incongruent with the studies in NZM2328.Lc4 mice that develop glomerular immune complex deposits and fatal lupus glomerulonephritis in the absence of antinuclear or anti-dsDNA antibody in serum or in IgG eluted from kidney homogenates, thereby demonstrating that antinuclear or anti-dsDNA antibodies are not essential for lupus glomerulonephritis [13].

The absolute requirement for anti-DNA or antinuclear antibodies in lupus glomerulonephritis is also challenged by studies from patient samples. Immunoglobulin eluted from kidney tissue obtained at autopsy from 25 lupus patients were screened against a panel of 14 different antigens including dsDNA, chromatin, collagen-like regions of C1q complement, Sm, SSA, SSB and histones [14]. A cumulative reactivity to all these antigens in the different samples ranged from 0.3 to $41.3 \%$ of the total IgG eluted from the respective kidneys. Thus, a large fraction of renal IgG reacts to unknown antigens. This is further complicated by potential crossreactivity to different antigens including binding to the blocking agents like bovine serum albumin, a notorious characteristic of lupus sera in ELISAs [15]. Indeed, glomerular proteome arrays show that serum from lupus mice and patients react with multiple antigens. Identification of antigen clusters from the glomerular proteome recognized by patient sera may be a better predictor of disease [16]. In another study [17••], reactivity of 200 monoclonal antibodies generated by isolating single $\mathrm{IgG}+$ memory B cells from four lupus patients and one healthy control showed that $15-26 \%$ of the antibodies were polyreactive between a diverse antigen panel that included ssDNA, dsDNA, LPS, and insulin. Surprisingly, there was no significant difference between the patients and controls in the autoreactivity of the memory IgG+ B cells, 
implying that polyreactive autoantibody populations are a common occurrence in all individuals. However, this can be explained by the increasing recognition that many $\mathrm{B}$ and $\mathrm{T}$ cell receptors are polyreactive [18].

The studies presented above suggest that development of lupus glomerulonephritis is not associated with pathogenic autoantibodies of singular specificities. In fact, majority of the antigens recognized by glomerular $\mathrm{IgG}$ deposits are unknown. In view of the recent findings that immune complexes per se are insufficient to cause end stage renal failure and that endorgan responses to these deposits are important factors for the progression of lupus nephritis, identifying specificities of autoantibodies that are deposited in glomeruli of lupus patients may not be critical for prognosis of renal disease.

\section{Autoreactive T cells and lupus glomerulonephritis}

Since autoantibodies of multiple specificities are a hallmark of SLE, it is natural that the role of B cells and autoantibodies has been a dominant focus of study in SLE in general and in lupus glomerulonephritis in particular. T cells were considered mainly for the provision of help to B cells for the production of autoantibodies. During the past several years, the focus on the role of $\mathrm{T}$ cells in lupus glomerulonephritis has been changed to the emphasis of these cells as effectors mediating tissue injury.

Evidence for the importance of $\mathrm{T}$ cells as effectors in lupus glomerulonephritis comes from several approaches. The first approach was the finding that renal disease is present in MRL/ lpr mice lacking circulating Ig but with B cells expressing a B cell receptor transgene [19]. The study showed that MRL/lpr mice expressing a surface transgenic B cell receptor to 4hydroxy-3-nitrophenyl develop unique renal disease characterized by glomerular sclerosis and interstitial inflammation despite the absence of circulating Ig. Secondly, NZB/W F1 mice with established chronic glomerulonephritis treated with CTLA4Ig and a suboptimal dose of cyclophosphamide showed a significant delay in mortality without reduction in glomerular immune complex deposits [20]. Thus, blocking T cell activation by CTLA4Ig could prevent disease progression. This reaffirms the original observation by Wofsy et al. [21] that anti-T cell antibody therapy of NZB/WF1 mice reduced glomerular inflammation, severe proteinuria and early mortality. In NZM2328 mice, early immune complex deposits and acute proliferative glomerulonephritis was associated with glomerular and peri-glomerular $\mathrm{T}$ cell infiltration [22]. In addition, MHC II positive, CD11c dendritic cells were seen in the glomeruli. This was accompanied by increased frequency of CD4+ $\mathrm{T}$ cell activation in the regional lymph nodes. All these findings suggest a local $\mathrm{T}$ cell response in kidney and regional lymph nodes early in the disease process.

Recently, using a transgenic mouse model system, Heymann et al. [23••] have demonstrated a role for CD4 and CD8 T cells in glomerular injury. Transgenic mice expressing ovalbumin and hen egg lysozyme proteins in glomerular epithelial cells, the podocytes, were generated. Ovalbumin-specific transgenic CD8+ T cells injected into these mice could get activated and expanded in the renal lymph nodes. The T cell activation was prevented by depletion of CD11c dendritic cells. These studies suggest that uptake of podocyte antigens by dendritic cells and cross-presentation to CD8+ T cells occurs in the renal lymph nodes. Transfer of ovalbuminspecific CD4+ T cells did not result in expansion or division. However, repeated co-injection of ovalbumin-specific activated CD4 and naïve CD8 T cells caused renal disease characterized by peri-glomerular inflammation, infiltration of macrophages and dendritic cells, and onset of mild proteinuria. Although this model does not completely mimic the regional CD4+ T cell activation in lupus mice, it is the first direct demonstration for the role of dendritic cells and $\mathrm{CD} 4+$ and CD8+ T cells in glomerular injury. Another significant difference of this model 
from lupus glomerulonephritis is the absence of glomerular immune complex deposits. These differences should be taken into account in its applicability to lupus nephritis.

Careful analysis of the kinetics of inflammatory cell migration and chemokine expression in the NZB/W F1 kidneys collected at different ages show distinct patterns of gene expression with disease progression [24••]. Cohorts of mice in remission following treatment with cyclophosphamide, CTLA4Ig, and anti-CD154 were also studied. A significant finding of this study was that infiltration of activated macrophages in the renal interstitium corresponded with disease progression. Significantly, remission following treatment was associated with reduced macrophage activation. Thus, macrophage infiltration is a key indicator of disease progression. As discussed earlier, peri-glomerular inflammation caused by glomerular antigen-reactive $\mathrm{T}$ cells in the renal cortex leads to increased macrophage infiltration. It is likely that the inflammatory macrophages are attracted to the renal interstitium as a result of the periglomerular $\mathrm{T}$ cell infiltrate.

Several recent studies have investigated the pathogenic capacity of different CD4+ T cell subsets. Kidneys of nephritic NZM2328 mice show increased expression of Th1 cytokines (IFN gamma and IL12) [22]. A recent study [25•] showed increased mortality in NZM2328 mice deficient in TNF receptor 1 and 2. The TNF receptor deficient mice had accelerated onset of renal disease with activated CD4+ cells showing a Th17 gene profile. Thus, in addition to Th1 T cells, lupus glomerulonephritis can also be mediated by Th17 T cells. Laser microdissection of glomerular, periglomerular and perivascular infiltrates in MRL/lpr mice also showed increased IL17 expression, along with IFN gamma and IL13 [26]. This is further supported by the study of Crispin et al. [27••] showing expanded double negative T cells producing IL17 and infiltrating kidneys in patients with SLE. Other studies suggesting a potential role of Th17 cells in SLE have been reviewed by Garrett-Sinha et al. [28]. The follicular helper T cell subsets characterized as IL21 producing CD4 + inducible T cell costimulator (ICOS) + cells have been identified in spleens of BXSB-ya $a$ mice [29•]. In another study [30•], MRL/lpr mice lacking ICOS show a significant reduction in IgG autoantibody, but a modest reduction in glomerulonephritis. The role of these subsets in renal disease is unclear.

In summary an increasing number of studies support a role for $\mathrm{T}$ cell mediated renal disease in SLE. The next advance in lupus nephritis is the identification of target antigens that activate autoreactive $\mathrm{T}$ cells leading to glomerular and interstitial infiltration of inflammatory cells.

\section{Pathogenic contribution of end-organ responses}

Glomerular immune complex deposition is one of the earliest signs of renal involvement in SLE. In MRL/lpr mice, this is followed by production of chemokines like MCP-1 and RANTES in the glomeruli, primarily by mesangial cells [31]. The initial mesangial cell stimulation leads to acute proliferative glomerulonephritis characterized by mesangial expansion and cellular infiltration into the glomeruli. This may progress to chronic glomerulonephritis characterized by glomerulosclerosis, interstitial fibrosis and tubular atrophy, along with severe persistent proteinuria and fatal renal failure. We have previously demonstrated that in NZM2328 mice, acute glomerulonephritis need not progress to chronic glomerulonephritis [32]. This lack of disease progression is also seen in a subset of patients [33]. To investigate molecular changes associated with progressive glomerular disease, microarray analyses for glomerular gene expression patterns at different stages of glomerulonephritis were carried out in NZM2328 mice [34]. Transcriptional profiles could be classified into four distinct clusters, that is normal, acute, transitional and chronic glomerulonephritis. Acute glomerulonephritis was dominated by inflammatory chemokines and cytokines and markers of myeloid cells. The chronic glomerulonephritis stage was characterized by glomerular fibrosis and sclerosis, with increase 
in tissue repair and remodeling genes. Severe proteinuria and progressive loss of renal function is associated with chronic glomerulonephritis. The micro-array analysis identified a transitional glomerulonephritis stage showing increased expression of seven genes including matrix metalopeptidase, Mmp10; transforming growth factor beta 2, TGF 2 ; insulin-like growth factor binding protein 2, igfbp 2; and lipocalin 2, Lcn2. Activation of TGF beta signaling appears to be the final common pathway leading to fibrosis and irreversible glomerular damage, resulting in end-stage renal disease [35]. This pathway is depicted in Fig. 1 and provides novel targets for therapeutic intervention.

Considerable interest has been focused on the role of Toll-like receptors (TLRs) in the autoimmune disease and has been reviewed recently [36•]. It is mentioned here to emphasize the importance of the TLR expression on renal cells and their role in activation of end organ responses in tissue injury.

\section{Genetic control of end-organ damage}

Murine models of spontaneous SLE have been used for identification of mapping of genes controlling susceptibility to SLE. Our studies in NZM2328, using (C57L/J $\times$ NZM2328)F1 $\times$ NZM2328 backcross mice identified unique genetic intervals dictating susceptibility to three distinct phenotypes: acute glomerulonephritis (Agnzl on chromosome 1 and Agnz2 on chromosome 17), chronic glomerulonephritis and proteinuria (Cgnzl on chromosome 1) and antinuclear and anti-dsDNA autoantibodies (Adazl on chromosome 4) [37]. Congenic mice were generated on an NZM2328 lupus-susceptible genetic background containing C57L/J lupus-resistant chromosomal segments on chromosomes 1 and 4 [13]. The NZM2328.C57Lc1 failed to develop autoantibodies or fatal glomerulonephritis, whereas NZM2328.C57Lc4 did not generate antinuclear or anti-dsDNA autoantibodies but died of severe lupus glomerulonephritis and renal failure. These phenotypes in the congenic mouse strains, NZM2328.C57Lc1 and NZM2328.C57Lc4, respectively, confirmed the initial genetic mapping studies. These studies show a clear dissociation of genetic susceptibility between antinuclear anti-dsDNA autoantibodies and lupus glomerulonephritis.

To identify genes controlling progression to chronic glomerulonephritis, new congenic lines were generated using an NZM2328.Lc1 × NZM2328 cross [38]. One of these lines, R27, contains an $8 \mathrm{Mb}$ C57L/J chromosome 1 fragment with the Cgnzl locus on an NZM2328 genetic background. The Agnzl locus was not replaced and is from the parental NZM2328 lupus-susceptible strain. The R27 mice failed to develop antinuclear and anti-dsDNA antibodies. All of the R27 mice developed glomerular immune complexes and acute proliferative glomerulonephritis. Significantly, all R27 mice survived for 12 months and did not develop renal failure. This provides genetic validation for the hypothesis that genetic control of acute glomerulonephritis and chronic glomerulonephritis are distinct. This further emphasizes the importance of organ resistance in determining the outcome of immune complex-mediated renal disease. Thus, acute glomerulonephritis need not progress to chronic glomerulonephritis. As depicted in Fig. 2, renal failure in SLE is a consequence of interaction between innate and adaptive immune responses dictated by genetic susceptibility.

Recent studies in the R27 mice show that genes for complement receptor 2 and Fc inhibitory receptors may not be crucial in end-organ damage ( $\mathrm{Ge}$ and $\mathrm{Fu}$, unpublished). The R27 region contains Ly108 identified as the lupus gene using B6.Sle1 mice [39]. Since the Sle1 on B6 background deals with autoantibody formation, the Ly108 is likely involved in the generation of antinucleosome antibodies. Other genes in the R27 region that may affect end organ responses are being studied.

The concept of end-organ resistance is also illustrated by the recent studies of Mohan and colleagues [40-42] using a murine model of immune complex glomerulonephritis. Immune 
complex glomerulonephritis was induced by immunizing mice with rabbit IgG in Complete Freunds' Adjuvant (CFA) followed by injection of rabbit anti mouse GBM serum in 20 different inbred mouse strains. Strains like NZW, DBA/1, 129/SvJ developed severe glomerulonephritis, whereas C57BL/6 and BALB/C mice did not. The severity of renal disease did not corelate with the strength of the immune response to rabbit IgG. These studies showed strain-dependent differences in renal disease and therefore potentially genetic control of susceptibility to kidney injury. Following disease induction, gene expression was studied in renal cortex of susceptible and resistant mouse strains [43••]. Of the 50 genes consistently down-regulated in the susceptible mice, 10 belonged to the kallikrein $(K l k)$ family. Kallikreins are serine esterases that are involved in multiple functions including regulation of inflammation, apoptosis, and renal fibrosis. Kallikreins stimulate production of bradykinins that bind bradykinin receptors, B1 and B2 to exert their biological effect. Blocking the bradykinin B2 receptor enhanced renal disease in resistant BALB/c mice and administration of bradykinin-reduced glomerulonephritis in susceptible $129 / \mathrm{SvJ}$ mice. The Klk gene complex is located on chromosome 7 within a lupus-susceptibility locus in the NZM2410 lupus mice. Sequencing of the differentially expressed $K l k$ genes did not show dissimilarities between resistant and susceptible strains. However, the promoter regions of five Klkl genes showed several single-nucleotide polymorphisms. In addition, the resistant strains had a 20-bp deletion in the $K l k b 3$ promoter. These studies support a role for Klk gene expression in the kidney dictating susceptibility to immune complex-mediated renal injury. Extension of these studies to patient cohorts shows KLK gene polymorphisms in some ethnic groups, specifically in patients of European descent.

\section{Conclusion}

The current concepts of lupus nephritis have important clinical implications. The realization of the importance of $\mathrm{T}$ cells as central players of this disease suggests that anti-T cell agents are required for inducing remission. The role inflammation as seen in the early proliferative nephritis can best be dealt with using anti-inflammatory reagents such as prednisone. The importance of cellular response to inflammatory signals by glomerular endothelial and mesangial cells identifies these cells as therapeutic targets. Recently, we and others have developed immunoliposomal systems for targeting drug delivery to renal glomeruli [44••,45]. The realization that end-organ resistance to damage is under genetic control indicates the need to personalize therapeutic strategies in the treatment of lupus nephritis to minimize side effects. It is also evident that prevention of flares in the maintenance phase of the disease requires immunomodulating therapy to reduce innate immune response and prevent activation of autoreactive $\mathrm{T}$ and $\mathrm{B}$ cells.

\section{Acknowledgments}

The work was supported by P50-AR045222 and R01-AR047988 grants from National Institute of Arthritis and Musculoskeletal and Skin Diseases to S.M.F; R01 DK069769 from National Institute Diabetes, Digestive and Kidney Disease to H.B; and grants from Alliance for Lupus Research Award \# 68164 (S.M.F.) and Award \#113300 (H.B.). The authors have no conflicts of interest.

\section{References and recommended reading}

Papers of particular interest, published within the annual period of review, have been highlighted as:

- of special interest

- $\quad$ •• of outstanding interest

Additional references related to this topic can also be found in the Current World Literature section in this issue (pp. 563-564). 
1. Lahita, RG. The clinical presentation of systemic lupus erythematosus. In: Lahita, RG., editor. Systemic lupus erythematosus. 3. San Deigo: Academic Press; 1999. p. 325-336.

2. Tan EM, Schur PH, Carr RI, Kunkel HG. Deoxybonucleic acid (DNA) and antibodies to DNA in the serum of patients with systemic lupus erythematosus. J Clin Invest 1966;45:1732-1740. [PubMed: 4959277]

3. Migliorini P, Baldini C, Rocchi V, Bombardieri S. Anti-Sm and anti-RNP antibodies. Autoimmunity 2005;38:47-54. [PubMed: 15804705]

4. Vlahakos DV, Foster MH, Adams S, et al. Anti-DNA antibodies form immune deposits at distinct glomerular and vascular sites. Kidney Int 1992;41:1690-1700. [PubMed: 1501424]

5. Deocharan B, Qing X, Lichauco J, Putterman C. Alpha-actinin is a cross-reactive renal target for pathogenic anti-DNA antibodies. J Immunol 2002;168:3072-3078. [PubMed: 11884481]

6. Foster MH, Sabbaga J, Line SR, et al. Molecular analysis of spontaneous nephrotropic antilaminin antibodies in an autoimmune MRL-lpr/lpr mouse. J Immunol 1993;151:814-824. [PubMed: 8335911]

7. van Bruggen MC, Kramers C, Walgreen B, et al. Nucleosomes and histones are present in glomerular deposits in human lupus nephritis. Nephrol Dial Transplant 1997;12:57-66. [PubMed: 9027774]

8. Zykova SN, Seredkina N, Benjaminsen J, Rekvig OP. Reduced fragmentation of apoptotic chromatin is associated with nephritis in lupus-prone (NZB x NZW)F(1) mice. Arthritis Rheum 2008;58:813825. [PubMed: 18311834]

9. Mortensen ES, Fenton KA, Rekvig OP. Lupus nephritis: the central role of nucleosomes revealed. Am J Pathol 2008;172:275-283. [PubMed: 18187568]

10. Mjelle JE, Kalaaji M, Rekvig OP. Exposure of chromatin and not high affinity for dsDNA determines the nephritogenic impact of anti-dsDNA antibodies in (NZBxNZW)F1 mice. Autoimmunity 2009;42:104-111. [PubMed: 19005880]

11. Kalaaji M, Mortensen E, Jorgensen L, et al. Nephritogenic lupus antibodies recognize glomerular basement membrane-associated chromatin fragments released from apoptotic intraglomerular cells. Am J Pathol 2006;168:1779-1792. [PubMed: 16723695]

12. Kalaaji M, Sturfelt G, Mjelle JE, et al. Critical comparative analyses of antialpha-actinin and glomerulus-bound antibodies in human and murine lupus nephritis. Arthritis Rheum 2006;54:914926. [PubMed: 16508974]

13. Waters ST, McDuffie M, Bagavant H, et al. Breaking tolerance to double stranded DNA, nucleosome, and other nuclear antigens is not required for the pathogenesis of lupus glomerulonephritis. J Exp Med 2004;199:255-264. [PubMed: 14718514]

14. Mannik M, Merrill CE, Stamps LD, Wener M. Multiple autoantibodies form the glomerular immune deposits in patients with systemic lupus erythematosus. J Rheumatol 2003;30:1495-1504. [PubMed: 12858447]

15. Mannik, M. Letter about Zhen et al. JCI eletters. 2006. http://www.jci.org/eletters/view/23587

16. Zhen QI, Xie C, Wu T, et al. Identification of autoantibody clusters that best predict lupus disease activity using glomerular proteome arrays. J Clin Invest 2005;115:3428-3439. [PubMed: 16322790]

17••. Mietzner B, Tsuiji M, Scheid J, et al. Autoreactive IgG memory antibodies in patients with systemic lupus erythematosus arise from nonreactive and polyreactive precursors. Proc Natl Acad Sci USA 2008;105:9727-9732. This study compares antigen-specificity of $\mathrm{IgG}+$ memory B cells in patients of lupus with healthy controls using single cell isolation and cloning of their B cell receptors. The strength of this study is the methodology for comparing B cell repertoires in lupus patients and normal individuals. [PubMed: 18621685]

18. Wucherpfennig KW, Allen PM, Celada F, et al. Polyspecificity of T cell and B cell receptor recognition. Semin Immunol 2007;19:216-224. [PubMed: 17398114]

19. Chan OT, Hannum LG, Haberman AM, et al. A novel mouse with B cells but lacking serum antibody reveals an antibody-independent role for B cells in murine lupus. J Exp Med 1999;189:1639-1648. [PubMed: 10330443]

20. Schiffer L, Sinha J, Wang X, et al. Short term administration of costimulatory blockade and cyclophosphamide induces remission of systemic lupus erythematosus nephritis in NZB/W F1 mice by a mechanism downstream of renal immune complex deposition. J Immunol 2003;171:489-497. [PubMed: 12817034] 
21. Wofsy D, Ledbetter JA, Hendler PL, Seaman WE. Treatment of murine lupus with monoclonal antiT cell antibody. J Immunol 1985;134:852-857. [PubMed: 3871218]

22. Bagavant H, Deshmukh US, Wang H, et al. Role for nephritogenic T cells in lupus glomerulonephritis: progression to renal failure is accompanied by $\mathrm{T}$ cell activation and expansion in regional lymph nodes. J Immunol 2006;177:8258-8265. [PubMed: 17114504]

23••. Heymann F, Meyer-Schwesinger C, Hamilton-Williams EE, et al. Kidney dendritic cell activation is required for progression of renal disease in a mouse model of glomerular injury. $\mathrm{J}$ Clin Invest 2009;119:1286-1297. This study uses a transgenic mouse system to demonstrate activation of glomerular antigen-specific $\mathrm{T}$ cells in the renal lymph nodes. The findings in this study can give some insight into pathogenic potential of autoreactive T cells in lupus nephritis. [PubMed: 19381017]

24••. Schiffer L, Bethunaickan R, Ramanujam M, et al. Activated renal macrophages are markers of disease onset and disease remission in lupus nephritis. J Immunol 2008;180:1938-1947. The study describes the disease of lupus glomerulonephritis by quantitative real-time PCR of chemokine and cytokine gene expression in renal cortex of NZB/W F1 mice at different stages of disease. This study proposed infiltration of activated renal macrophages as the harbingers of disease progression in lupus nephritis. [PubMed: 18209092]

25•. Jacob N, Yang H, Pricop L, et al. Accelerated pathological and clinical + nephritis in systemic lupus erythematosus-prone New Zealand mixed 2328 mice doubly deficient in TNF receptor I and TNF receptor 2 via a TH-17 associated pathway. J Immunol 2009;182:2532-2541. This study investigates the mechanism of accelerated renal disease in mice lacking both TNF receptors 1 and 2. [PubMed: 19201910]

26. Wang Y, Ito S, Chino Y, et al. Use of laser microdissection in the analysis of renal-infiltrating T cells in MRL/lpr mice. Mod Rheumatol 2008;18:385-393. [PubMed: 18452052]

27••. Crispin JC, Oukka M, Bayliss G, et al. Expanded double negative T cells in patients with systemic lupus erythematosus produce IL-17 and infiltrate the kidneys. J Immunol 2008;181:8761-8766. This is the first study demonstrating TH $17 \mathrm{~T}$ cells in renal disease in lupus patients. [PubMed: 19050297]

28. Garrett-Sinha LA, John S, Gaffen SL. IL-17 and the Th17 lineage in systemic lupus erythematosus. Curr Opin Rheumatol 2008;20:519-525. [PubMed: 18698171]

29•. Bubier JA, Sproule TJ, Foreman O, et al. A critical role for IL-21 receptor signaling in the pathogenesis of systemic lupus erythematosus in BXSB-Yaa mice. Proc Natl Acad Sci U S A 2009;106:1518-1523. This study investigates the possible role of T follicular helper cells in murine models of lupus glomerulonephritis. [PubMed: 19164519]

30•. Odegard JM, DiPlacido LD, Greenwald L, et al. ICOS controls effector function but not trafficking receptor expression of kidney-infiltrating effector T cells in murine lupus. J Immunol 2009;182:4076-4084. This study investigates the role of T cell infiltration in the kidney as a critical factor for renal disease in lupus-susceptible mice. [PubMed: 19299705]

31. Perez DL, Maier H, Nieto E, et al. Chemokine expression precedes inflammatory cell infiltration and chemokine receptor and cytokine expression during the initiation of murine lupus nephritis. J Am Soc Nephrol 2001;12:1369-1382. [PubMed: 11423566]

32. Bagavant H, Tung KS. Failure of CD25+ T cells from lupus-prone mice to suppress lupus glomerulonephritis and sialoadenitis. J Immunol 2005;175:944-950. [PubMed: 16002693]

33. Pollack P, Pirani C. Renal histologic findings in systemic lupus erythematosus. Mayo Clin Proc 1969;44:630-644. [PubMed: 5821138]

34. Wang H, Bagavant H, Deshmukh U, et al. Glomerular transcriptional profiles reveal the candidate biomarkers diagnostic for the progression of lupus nephritis from acute to chronic stages. Arthritis Rheum 2008;58:S317.

35. De Albuquerque DA, Saxena V, Adams DE, et al. An ACE inhibitor reduces Th2 cytokines and TGFbeta1 and TGF-beta2 isoforms in murine lupus nephritis. Kidney Int 2004;65:846-859. [PubMed: 14871404]

36•. Allam R, Anders HJ. The role of innate immunity in autoimmune tissue injury. Curr Opin Rheumatol 2008;20:538-544. This study summarizes recent literature on the TLRs and autoimmunity. [PubMed: 18698174] 
37. Waters ST, Fu SM, Gaskin F, et al. NZM2328: a new mouse model of systemic lupus erythematosus with unique genetic susceptibility loci. Clin Immunol 2001;100:372-383. [PubMed: 11513551]

38. Ge Y, Jiang C, Morris A, et al. Acute glomerulonephritis without progression to chronic glomerulonephritis, renal failure and early Mortality in female mice of congenic strain NZM2328. Lc1R27. Arthritis Rheum 2008;58:S441.

39. Kumar KR, Li L, Yan M, et al. Regulation of B cell tolerance by the lupus susceptibility gene Ly108. Science 2006;312:1665-1669. [PubMed: 16778059]

40. Xie C, Zhou XJ, Liu X, Mohan C. Enhanced susceptibility to end-organ disease in the lupusfacilitating NZW mouse strain. Arthritis Rheum 2003;48:1080-1092. [PubMed: 12687552]

41. Xie C, Sharma R, Wang H, et al. Strain distribution pattern of susceptibility to immune-mediated nephritis. J Immunol 2004;172:5047-5055. [PubMed: 15067087]

42. Xie C, Rahman ZS, Xie S, et al. Strain distribution pattern of immune nephritis: a follow-up study. Int Immunol 2008;20:719-728. [PubMed: 18381352]

43••. Liu K, Li QZ, Delgado-Vega AM, et al. Kallikrein genes are associated with lupus and glomerular basement membrane-specific antibody-induced nephritis in mice and humans. J Clin Invest 2009;119:911-923. This study demonstrates that renal expression of the kallekrein genes can influence immune complex-mediated glomerulonephritis. This study clearly implicates the contribution of end-organ gene expression in determining outcome of immune complex-mediated renal injury. The hypothesis based on mouse studies has been tested in cohorts of lupus patients. [PubMed: 19307730]

44••. Scindia Y, Deshmukh U, Thimmalapura PR, Bagavant H. Antialpha8 integrin immunoliposomes in glomeruli of lupus-susceptible mice: a novel system for delivery of therapeutic agents to the renal glomerulus in systemic lupus erythematosus. Arthritis Rheum 2008;58:3884-3891. This study describes the first successful demonstration of strategy for delivery of agents preferentially to the mesangium and mesangial cells of mouse glomeruli following tail vein injection. This technology can be adapted for use in humans. [PubMed: 19035491]

45. Asgeirsdottir SA, Zwiers PJ, Morselt HW, et al. Inhibition of proinflammatory genes in anti-GBM glomerulonephritis by targeted dexamethasone-loaded AbEsel liposomes. Am J Physiol Renal Physiol 2008;294:F554-F561. [PubMed: 18160627] 


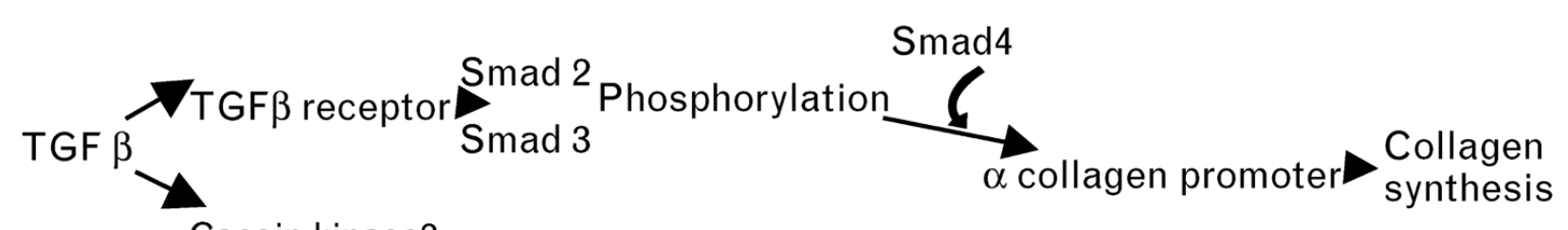

Caesin kinase2

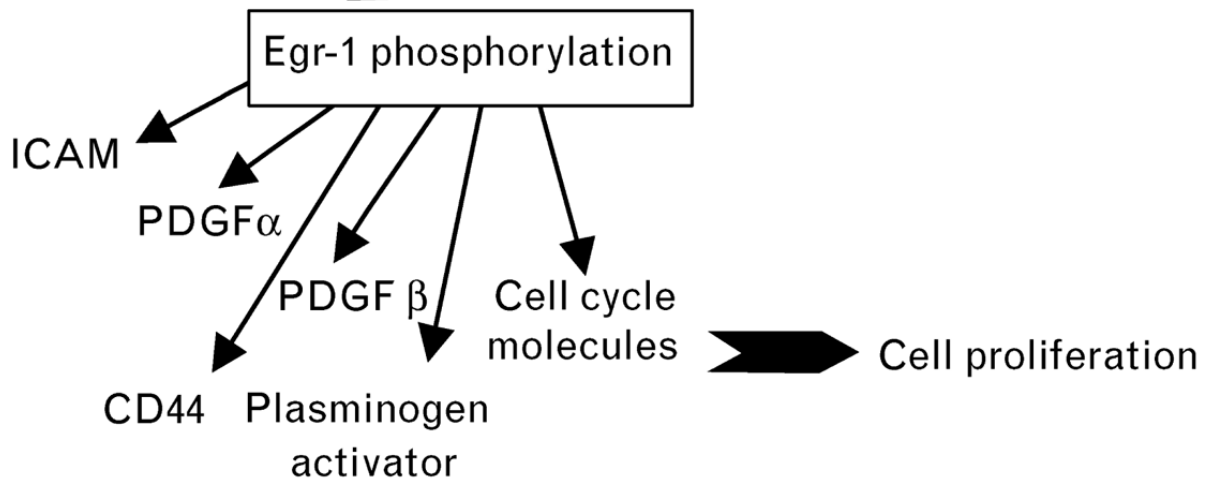

Figure 1. A schematic showing TGF beta signaling pathway and its interaction with other molecules like PDGF, CD44 and cyclins through transcription factor Egr-1

PDGF, platelet-derived growth factor. 


\section{Susceptible host}

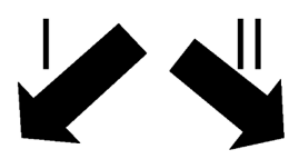

Hyper activation of genes important in the innate and adaptive immune systems leading to the unregulation of cytokine gene expression and lowering of the activation threshold of immune cells

Genes controlling end organ susceptibility to damage
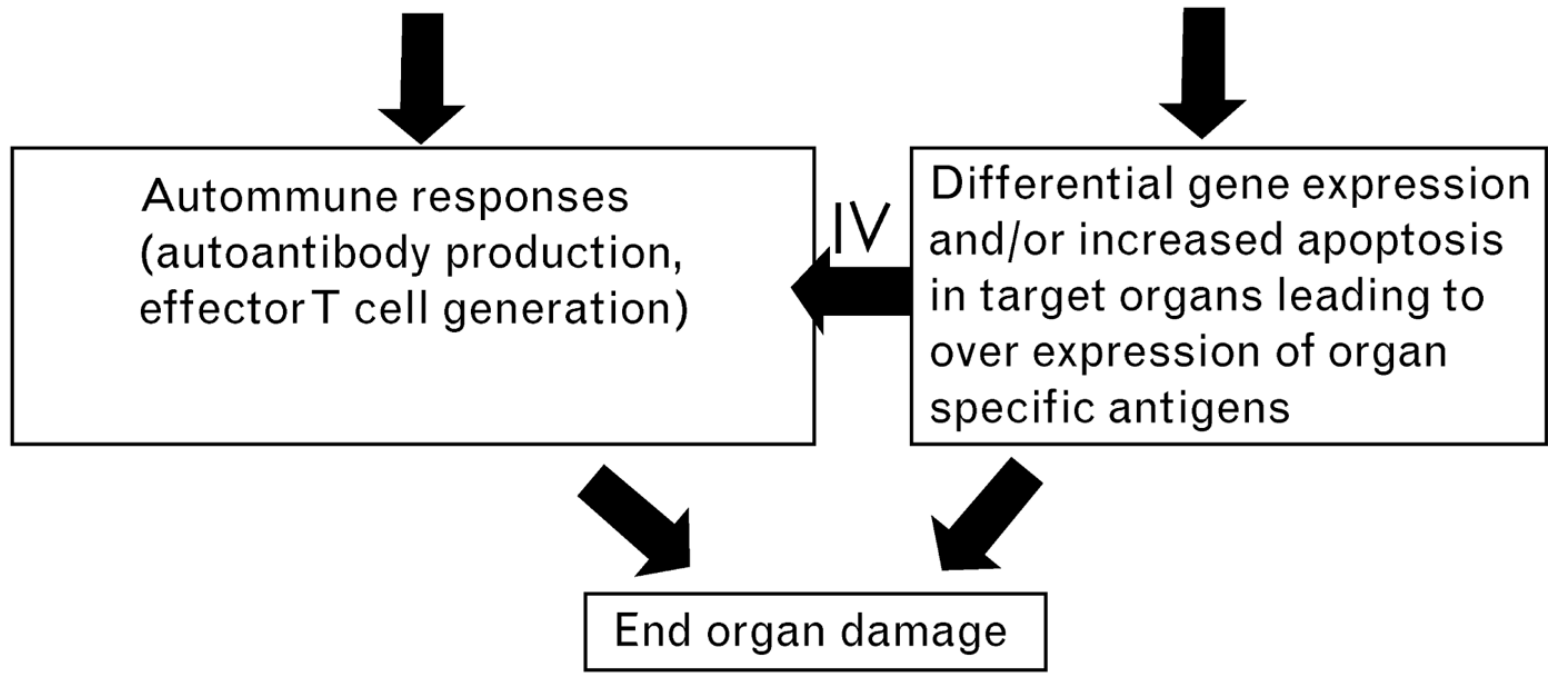

Figure 2. Renal disease in SLE is determined by interaction between innate and adaptive immune responses and genetic susceptibility of the end organ to injury

SLE, systemic lupus erythematosus. The figure was previously published in the study by Waters et al. [13]. 